\title{
POPULATION

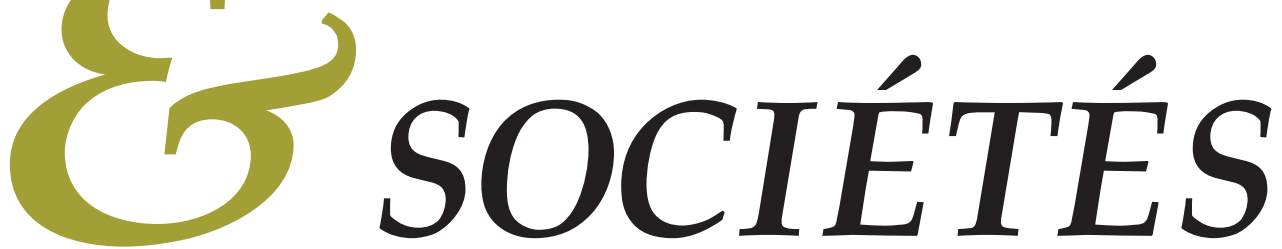

\section{Le Japon face au déclin annoncé de sa population}

\author{
Jacques Véron*
}

\begin{abstract}
Le monde développé vit avec la crainte du vieillissement démographique et de la dépopulation, les adultes ayant dans beaucoup de pays moins d'enfants qu'il n'en faudrait pour remplacer les générations. Avec l'une des plus basses fécondités du monde -1,3 enfant en moyenne par femme- et la durée de vie la plus élevée, le Japon est exemplaire de cette évolution. S'appuyant sur les dernières projections de population japonaise, Jacques Véron nous explique les certitudes et les interrogations quant à son avenir démographique.
\end{abstract}

L es projections démographiques établies fin 2006 par l'Institut national de recherche japonais sur la population et la sécurité sociale annoncent un effectif de l'ordre de 95 millions d'habitants en 2050 pour la population du Japon, soit 32 millions de moins que les 128 millions de 2004 [1, 2]. Cette réduction s'explique par une très basse fécondité: d'après l'hypothèse centrale, le nombre moyen d'enfants par femme continuerait de diminuer dans les prochaines années puis remonterait très légèrement pour atteindre 1,26 en 2055. À plus long terme la population diminuerait de plus de moitié, atteignant moins de 48 millions d'habitants en 2100 , toujours d'après la variante moyenne. Reflet de l'inquiétude quant à l'avenir, les projections, qui ont été poursuivies au-delà, annoncent l'extinction totale de la population japonaise autour de l'an 3000 si la fécondité se maintenait indéfiniment basse. Si l'on en reste aux 50 prochaines années, cette décroissance annoncée de la population japonaise s'accompagnera d'une transformation très profonde de sa structure par âge et d'une réduction marquée de la population active.

\section{Une fécondité longtemps en baisse} et l'espérance de vie la plus haute du monde

Après un baby-boom extrêmement court (pas plus de trois ans), la fécondité japonaise a beaucoup baissé dans l'après-guerre, de 3,65 enfants par femme en 1950 à 2,04 en 1959, puis a remonté à la fin des années 1960 et au

* Institut national d'études démographiques
Figure 1 - Évolution de la fécondité japonaise, de 1950 à 2007

Nombre moyen d'enfants par femme

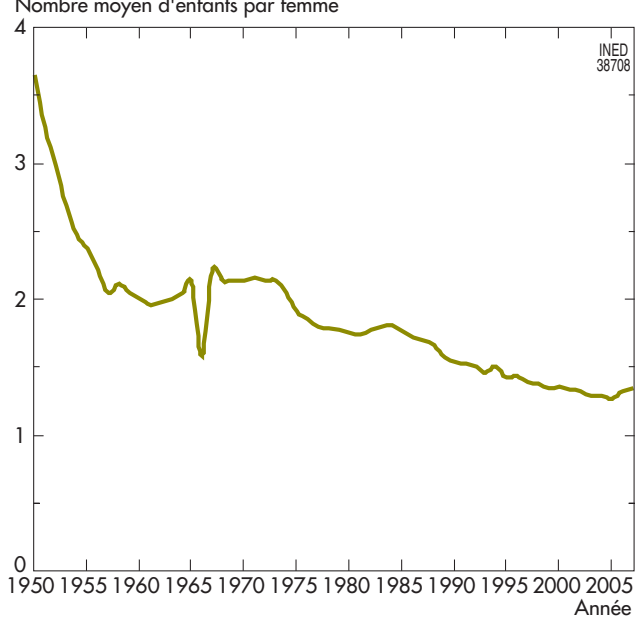

Note: L'année 1966 est une année «cheval et feu» de l'horoscope chinois (voir texte).

Source: [1]

(Jacques Véron, Population \& Sociétés n 449, Ined, octobre 2008)

tout début des années 1970 au-dessus du niveau de remplacement des générations (figure 1). En 1966, la fécondité chute exceptionnellement à 1,58 enfant en raison de la combinaison astrologique de l'année 1966, année "cheval et feu» pour l'horoscope chinois. Selon une croyance répandue, les filles nées une telle année porteraient malheur à leur mari. Certains parents ont donc préféré retarder la naissance d'un enfant ou déclarer la naissance en 1965 ou 1967 [3]. Repartie à la baisse à partir de 1974, la fécondité n'a cessé depuis de décroître. 
Lorsqu'en 1990 la fécondité se rapproche de la valeur de 1,5 enfant par femme observée en 1966, la société japonaise semble subir un choc, dénommé «1.53 shock», lié à la prise de conscience des changements à l'œuvre, en particulier la diminution du nombre de jeunes travailleurs et le vieillissement de la population [4]. La fécondité n'en poursuit pas moins sa baisse dans les quinze années suivantes et le nombre moyen d'enfants par femme chute à 1,26 en 2005. La fécondité remonte un peu en $2006(1,32)$ et en $2007(1,34)$.

$\mathrm{Au}$ cours des cinquante dernières années, l'âge moyen des femmes à la maternité, tous rangs de naissance confondus, n'augmente que d'une seule année, passant de 28,9 ans en 1955 à 29,9 ans en 2004, mais la naissance du premier enfant est de plus en plus tardive (28,5 ans aujourd'hui contre 25,1 ans au milieu des années 1950). Dans une société où les naissances hors mariage restent très rares -elles représentent moins de $2 \%$ des naissances en 2004- le retard du mariage et la montée du célibat féminin ont entraîné mécaniquement une baisse de la fécondité (1). Entre 1950 et 2000, les proportions de femmes ayant entre 20 et 40 ans toujours célibataires progressent très fortement (tableau 1). Ainsi, $59 \%$ des femmes de 25-29 ans sont toujours célibataires en 2005 contre $15 \%$ en 1950. La proportion de célibataires parmi les femmes de 30-34 ans fait, pour sa part, plus que quintupler au cours de ces quelque cinquante années, passant de moins de $6 \%$ à $32 \%$.

Dans le même temps, la mortalité ne cesse de se réduire (tableau 2). L'espérance de vie à la naissance progresse de près de 20 ans chez les hommes entre le début des années 1950 et 2007, et de 23 ans chez les femmes. Atteignant 86,0 ans en 2007, l'espérance de vie des Japonaises est la plus élevée du monde. L'écart de durée de vie entre hommes et femmes s'accroît de manière marquée, doublant entre le début des années 1950 et 2007 (de 3,4 à 6,8 ans). L'espérance de vie à 65 ans progresse également de près de 7 années pour les hommes et de 10 années pour les femmes entre le début des années 1950 et 2005. À cet âge, il reste aux hommes 18,1 années à vivre en moyenne, et aux femmes, 23,2 années.

Cette baisse de la mortalité se traduit par une augmentation très rapide du nombre de centenaires: le Japon en comptait moins de 1000 en 1980 mais plus de 32000 en 2007. Plus de $85 \%$ des centenaires sont des femmes.

En 1950, les naissances étaient 2,6 fois plus nombreuses que les décès; en 2006, elles étaient à peu près en même nombre. On anticipe qu'en 2050, les décès seraient 2,4 fois plus nombreux que les naissances.

\section{Moins de familles élargies, plus de personnes vivant seules}

La baisse de la mortalité s'accompagne d'une forte progression de la proportion de ménages comptant une personne de 65 ans ou plus : leur nombre est multiplié

(1) On pourrait imaginer un effet en sens inverse: le désir d'enfant aurait diminué, entraînant du coup un moindre attrait du mariage, mais les enquêtes montrent que ce ne semble pas être le cas, les jeunes adultes souhaitant toujours avoir des enfants.
Tableau 1 - Proportions de femmes célibataires parmi les femmes ayant entre 20 et 40 ans en 1950 et en 2005 (en \%)

\begin{tabular}{|c|c|c|}
\hline \multirow{2}{*}{ Groupe d'âges } & 1950 & 2005 \\
\cline { 2 - 3 } & 55,3 & 88,7 \\
\hline $20-24$ ans & 15,2 & 59,0 \\
$25-29$ ans & 5,7 & 32,0 \\
$30-34$ ans & 3,0 & 18,4 \\
35-39 ans & \multicolumn{2}{|c|}{} \\
\hline Sources : [1] et [2] & (Jacques Véron, Population \& Sociétés n $^{\circ}$ 449, Ined, octobre 2008) \\
\hline
\end{tabular}

Tableau 2 - Évolution de l'espérance de vie à la naissance des hommes et des femmes au Japon de 1950 à 2007 (en années)

\begin{tabular}{|c|c|c|}
\hline & Hommes & Femmes \\
\hline $1950-1952$ & 59,6 & 63,0 \\
1960 & 65,3 & 70,2 \\
1970 & 69,3 & 74,7 \\
1980 & 73,3 & 78,8 \\
1990 & 75,9 & 81,9 \\
2000 & 77,7 & 84,6 \\
2007 & 79,2 & 86,0 \\
\hline Source : [2] & (Jacques Véron, Population \& Sociétés $\mathrm{n}^{\circ}$ 449, Ined, octobre 2008) \\
\hline
\end{tabular}

par 2,5 entre 1975 et 2004. En 1975, ces ménages sont majoritairement des familles à trois générations (54\% des cas). Il en va différemment aujourd'hui, la proportion de familles à trois générations dans les ménages comptant au moins une personne de plus de 65 ans n'est plus que de $21 \%$ en 2006. Au lieu de baisser, elle aurait pu s'élever, la réduction du nombre d'enfants et l'allongement de la vie augmentant a priori les chances pour un enfant de vivre dans une famille à trois générations [5]. On peut avancer l'idée que la famille à trois générations « fonctionne » de moins en moins bien, en particulier pour ce qui est de la répartition des rôles, lorsque les chances pour les grands-parents d'atteindre des âges élevés ne cessent d'augmenter. On peut aussi invoquer l'habitat urbain, qui rend difficile la cohabitation entre trois générations.

\section{Bouleversement de la structure par âge et diminution de l'offre de travail}

Si la population du Japon décroît conformément au scénario retenu dans la variante moyenne des projections de 2006, la dépopulation s'accompagnera d'un bouleversement de la structure par âge (figure 2). La part des plus de 65 ans dans la population totale, qui est de moins de $5 \%$ en 1950 et de $25 \%$ en 2007 , pourrait atteindre $32 \%$ en 2030 et près de $40 \%$ en 2050 (figure 3). Quant aux personnes de 75 ans ou plus, comptant pour à peine plus de $1 \%$ en 1950, elles pourraient représenter plus du cinquième de la population japonaise en 2050.

Le taux de dépendance démographique (2), après avoir connu une longue période de diminution de $72 \%$

(2) Rapport entre le nombre de personnes de moins de 15 ans ou de plus de 65 ans et celui de personnes de 15-64 ans. 
Figure 2 - Pyramide des âges du Japon au $1^{\text {er }}$ janvier 2007 et en 2055 (projection)

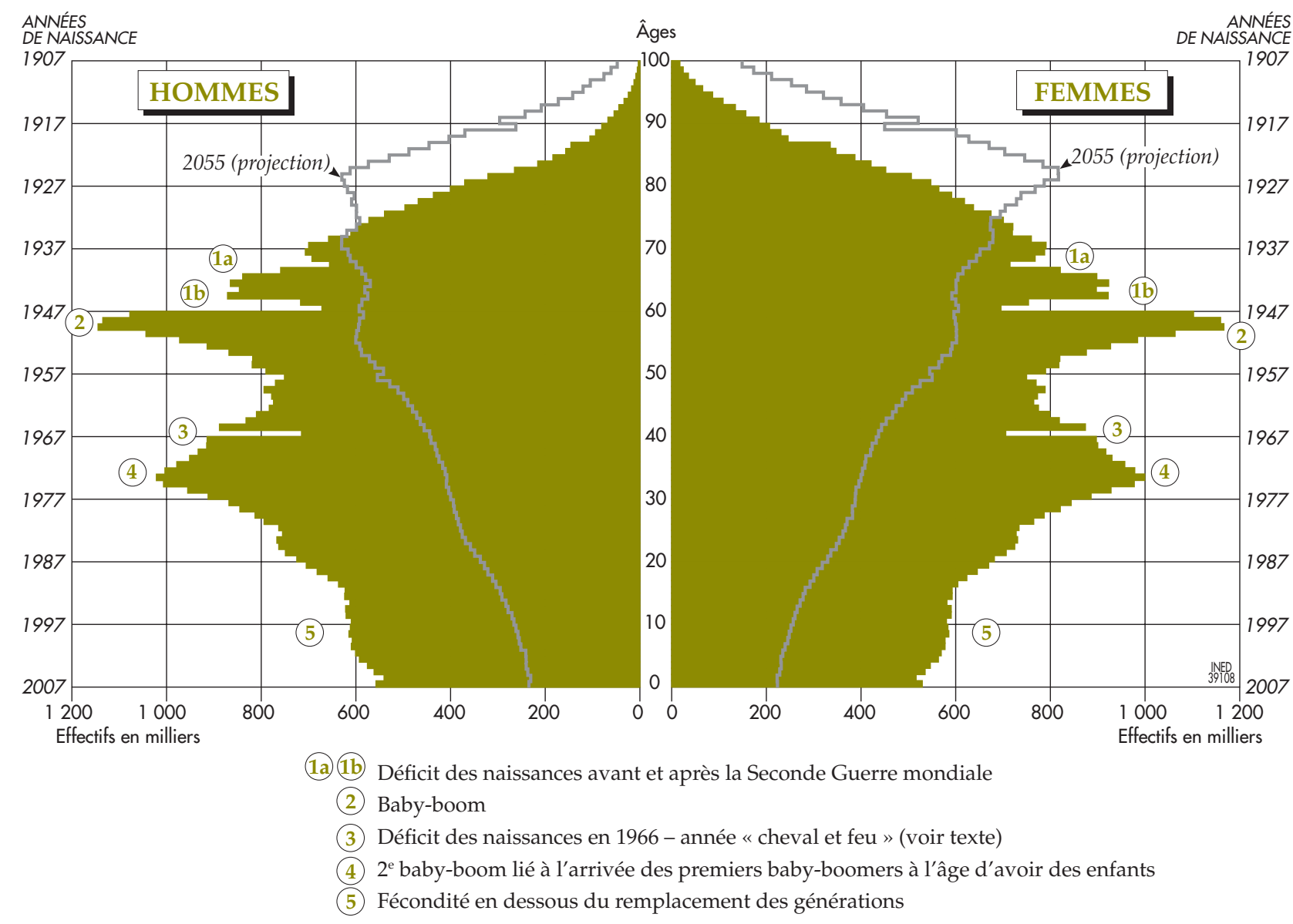

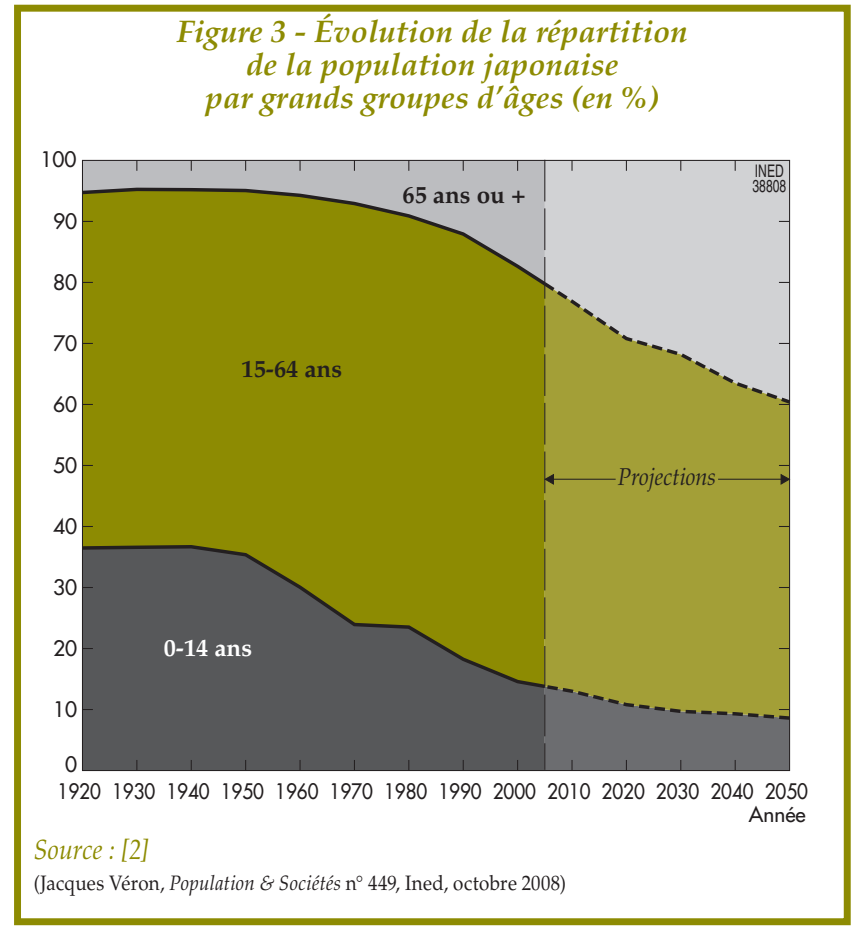

en 1920 à $43 \%$ en 1990, est remonté à $54 \%$ en 2007. Il pourrait progresser très fortement dans l'avenir et atteindre, d'après la variante moyenne des projections de 2006 qui, rappelons-le, se fonde sur une très basse fécondité, 93\% en 2050. Le taux de dépendance des jeunes ne varierait guère tandis que celui des plus de 65 ans ferait plus que doubler d'ici 2050. Entre 2000 et 2050, le nombre de plus de 65 ans pour 100 jeunes de moins de 15 ans s'accroîtrait très fortement, de 119 à 458.

La population active - population employée ou à la recherche d'un emploi - dont l'effectif double entre 1920 et la fin des années 1960, période à laquelle elle excède 52 millions, augmente encore fortement dans les trois décennies qui suivent et le nombre d'actifs dépasse 67 millions en 2001. À partir de cette date l'offre de travail se réduit et les projections tablent sur une poursuite de cette décroissance: en 2025, le Japon compterait environ 63 millions d'actifs. Au cours des trente dernières années, le taux global d'activité a diminué sensiblement, en raison de la tendance à quitter plus tôt le marché du travail, particulièrement pour les hommes. Si l'activité des femmes progresse, l'évolution de l'activité par âge témoigne de la persistance d'un cycle traditionnel d'activité (figure 4). Entre les âges de 35 et 55 ans les femmes sont certes de plus en plus fréquemment actives, mais la courbe d'activité n'en conserve pas moins son allure bimodale. Les projections de population active réalisées à l'horizon 2025 n'envisagent pas de modification importante des comportements d'activité féminine, même si les taux d'activité aux âges intermédiaires sont supposés poursuivre leur progression. 


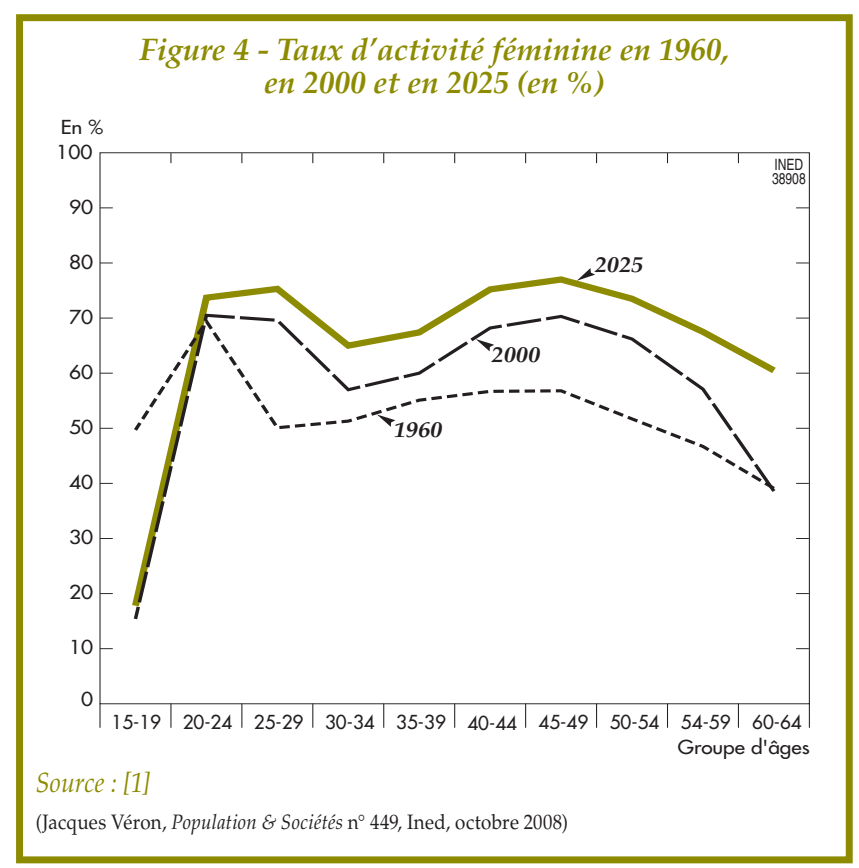

\section{Des évolutions inéluctables?}

La basse fécondité entraîne une diminution de la population; elle conjugue ses effets avec la basse mortalité pour induire un intense vieillissement démographique. Mais dans quelle mesure ces conséquences sont-elles inéluctables?

Si la mortalité ne variait plus à partir de 2006 et que la fécondité remontait brusquement au niveau du remplacement des générations cette même année pour s'y maintenir ensuite, la population du Japon connaîtrait un accroissement momentané, puis une réduction, et se stabiliserait, à partir des années 2070, autour d'un effectif de 110 millions d'habitants. Une telle remontée n'est évidemment pas réaliste, même si des enquêtes lors du « 1.53 shock» montraient que les Japonais continuaient de désirer plus de 2,1 enfants et considéraient que, pour réaliser leur désir d'enfant, il fallait rendre l'environnement plus favorable à l'enfant, réduire le coût économique de celui-ci, améliorer les conditions de logement et développer les aides comme les congés parentaux [4]. Dans son analyse du rôle joué par un

\section{RÉFÉRENCES}

[1] National Institute of Population and Social Security Research - Population Statistics of Japan 2008, http://www.ipss. go.jp/index-e.html

[2] Statistics Bureau - Japan Statistical Yearbook 2008, http:// www.stat.go.jp/english/data/nenkan/index.htm

[3] Biraben J.-N. - 1968, "L'année "Cheval et Feu"», Population, $\mathrm{n}^{\circ} 1$, pp. 154-162.

[4] YanAgishita M. - 1992, «Japan's declining fertility: "1.53 shock" ", Population Today, Apr; 20(4):3-4.

[5] Martin L. C. et Culter S. - 1983, «Mortality Decline and Japanese Family Structure» Population and Development Review $9, \mathrm{n}^{\circ} 4$, p. 633-649.

[6] Атон M. - 2001, «Very Low Fertility in Japan and Value Change Hypotheses", Review of Population and Social Policy, $\mathrm{n}^{\circ} 10,1-21$. changement des valeurs sur la fécondité, Makoto Atoh [6] note, à partir d'une série d'enquêtes, un changement de vues - devenues plus libérales - sur la sexualité, le mariage (une femme ne devant par exemple pas nécessairement se marier) et le divorce, mais il ne perçoit aucun signe d'une progression de l'individualisme depuis le milieu des années 1970. La baisse de la fécondité ne tiendrait pas à un quelconque refus de l'enfant, mais à un changement d'attitude à l'égard des relations parents-enfants, des relations conjugales et plus généralement des relations hommes-femmes [6]. Le mariage tardif, qui entraîne mécaniquement une basse fécondité, s'expliquerait avant tout par un changement du rôle et du statut des femmes. A priori, rien ne permet donc d'exclure une remontée de la fécondité. La très légère remontée des années 2006 et 2007 annoncerait-elle une reprise?

Si les évolutions démographiques sont conformes aux projections récentes, les effets sur l'offre de travail peuvent-ils être compensés par une modification des comportements d'activité? Les marges de manœuvre restent d'autant plus limitées que les projections supposent déjà un accroissement des taux d'activité des 60 64 ans. Pour ce qui est du groupe d'âges 65 ans ou plus, il est difficile d'envisager un scénario très différent de celui retenu dans les projections de population active. Mais si, en 2025, le taux global d'activité des femmes était le même que celui des hommes (71\% au lieu de $47 \%$ ), le nombre de femmes actives serait plus élevé de 1,35 million ; cela n'empêcherait toutefois pas la décroissance de la population active.

Il reste enfin le recours à la migration. Le Japon compte un peu plus de 2 millions d'étrangers enregistrés en 2006, dont 29\% de Coréens, 27\% de Chinois, $15 \%$ de Brésiliens et $9 \%$ de Philippins. Le gouvernement japonais envisage de mettre en place une politique d'immigration moins restrictive que celle en vigueur aujourd'hui mais, comme il ne semble être nullement question d'un recours massif à l'immigration, les conséquences démographiques resteront limitées.

Le Japon va donc devoir s'adapter à la décroissance de sa population, mais c'est le bouleversement de sa structure par âge qui constitue sans nul doute le défi majeur.

\section{RÉSUMÉ}

Les projections démographiques établies en 2006 par l'Institut national de recherche japonais sur la population et la sécurité sociale annoncent une réduction de la population japonaise de plus de 30 millions d'ici 2050 dans leur scénario central. La population japonaise devrait en outre vieillir fortement en raison de l'allongement de la durée de vie, déjà la plus élevée du monde, et de la très basse fécondité : 1,3 enfant en moyenne par femme en 2006. La population active continuerait de diminuer et le taux de dépendance s'accroîtrait fortement en raison de l'augmentation rapide de la proportion des 65 ans ou plus Une remontée limitée de la fécondité, une ouverture très mesurée des frontières ou une modification à la marge des comportements d'activité ne sont pas de nature à modifier radicalement les évolutions projetées. 